\title{
Impaired Sequence Learning in Carriers of the DYT1 Dystonia Mutation
}

\author{
Maria-Felice Ghilardi, MD, ${ }^{1,2}$ Maren Carbon, MD, ${ }^{3}$ Giulia Silvestri, MD, ${ }^{1}$ Vijay Dhawan, $\mathrm{PhD},{ }^{3,4}$ \\ Michele Tagliati, MD, ${ }^{4}$ Susan Bressman, MD, ${ }^{5}$ Claude Ghez, MD, ${ }^{1}$ and David Eidelberg, $\mathrm{MD}^{3,4}$
}

\begin{abstract}
Previous positron emission tomography (PET) studies have shown that nonmanifesting carriers of the DYT1 dystonia mutation express an abnormal pattern of resting glucose metabolism. To determine whether motor behavior is impaired in these subjects, we compared movement and sequence learning in 12 clinically unaffected DYT1 carriers with 12 age-matched controls. Regional differences in brain function during task performance were assessed with simultaneous $\mathrm{H}_{2}{ }^{15} \mathrm{O} / \mathrm{PET}$. We found that motor performance was similar in the DYT1 and control groups, with no significant differences in movement time and spatial accuracy measured during each of the tasks. In contrast, sequence learning was reduced in gene carriers relative to controls $(p<0.01)$. PET imaging during motor execution showed increased activation in gene carriers $(p<0.001$, uncorrected) in the left premotor cortex and right supplementary motor area, with concomitant reduction in the posterior medial cerebellum. During sequence learning, activation responses in DYT1 carriers were increased in the left ventral prefrontal cortex, and lateral cerebellum. These findings suggest that abnormalities in motor behavior and brain function exist in clinically nonmanifesting DYT1 carriers. Although localized increases in neural activity may enable normal movement execution in these subjects, this mechanism may not compensate for their defect in sequence learning.
\end{abstract}

Ann Neurol 2003;54:102-109

Primary torsion dystonia is a hyperkinetic movement disorder associated with several genetic variants. ${ }^{1}$ One genetic variant is the GAG deletion in the DYT1 gene at 9q34. This mutation commonly causes early limb onset primary torsion dystonia and is inherited as an autosomal dominant condition with incomplete (30-40\%) clinical penetrance. ${ }^{2}$ Using ${ }^{18} \mathrm{~F}$-fluorodeoxyglucose and positron emission tomography (PET), we had identified previously abnormalities in resting glucose utilization in the putamen/globus pallidus, supplementary motor area (SMA), and cerebellum of DYT1 mutation carriers, including clinically nonpenetrant subjects in whom signs and symptoms of dystonia are lacking. 3,4 Given the presence of metabolic abnormalities in key elements of motor control pathways, we sought to determine whether clinically unaffected mutation carriers also display abnormalities in the trajectory control of reaching movements and in learning motor sequences. To this end, we utilized a series of tasks with similar and welldefined kinematics to assess performance during motor execution and learning. ${ }^{5,6}$ Simultaneous PET recordings were acquired during task performance to determine whether specific abnormalities in brain activation re- sponses were present within corticostriatopallidothalamocortical loops and related pathways. ${ }^{7}$

\section{Subjects and Methods \\ Subjects}

We studied 12 right-handed nonmanifesting carriers of the DYT1 mutation (four men and eight women; aged $50.3 \pm$ 14.4 years, mean \pm standard deviation $[S D])$. These subjects were recruited through the Dystonia Clinical Research Center at Beth Israel Hospital in New York. A separate group of 12 right-handed healthy volunteer subjects (five men and seven women; aged $48.4 \pm 12.9$ years) served as controls. The subjects were recruited by advertisement among North Shore University Hospital and Columbia University personnel. This control group was demographically representative of these communities (including 25\% nonwhite participation), and the inadvertent inclusion of DYT1 mutation carriers was unlikely by chance. Written informed consent was obtained from all participants under protocols approved by the institutional review boards of the participating institutions.

The following exclusion criteria were applied to both groups: (1) history of neurological or psychiatric illness; (2) prior exposure to neuroleptic agents or drug use; (3) medical
From the ${ }^{1}$ Center for Neurobiology and Behavior, Columbia College of Physicians and Surgeons, New York, NY; ${ }^{2}$ INB-CNR, Milan, Italy; ${ }^{3}$ Center for Neurosciences, North Shore-Long Island Jewish Research Institute, Manhasset, NY; ${ }^{4}$ Department of Neurology, North Shore University Hospital, Manhasset, and New York University School of Medicine, New York; and ${ }^{5}$ Department of Neurology, Philips Ambulatory Care Center, Beth Israel Medical Center, New York, NY.
Received Oct 9, 2002, and in revised form Mar 13, 2003. Accepted for publication Mar 19, 2003.

Address correspondence to Dr Eidelberg, Center for Neurosciences, North Shore-Long Island Jewish Research Institute, 350 Community Drive, Manhasset, NY 11030. E-mail: david1@nshs.edu 
history of hypertension, cardiovascular disease, or diabetes mellitus; and (4) abnormal neurological examination. All subjects scored above 27 on Mini-Mental Examination and underwent magnetic resonance imaging to exclude structural brain abnormalities. All subjects performed the motor tasks described below. In addition, seven members of each group were scanned with ${ }^{15} \mathrm{O}$-labeled water $\left(\mathrm{H}_{2}{ }^{15} \mathrm{O}\right)$ and PET to measure brain activation responses during task performance.

\section{Motor Tasks}

The general features of our experimental paradigms have been presented in detail elsewhere. ${ }^{5,6}$ In these motor tasks, subjects moved a cursor on a digitizing tablet with their dominant right hand; cursor and target positions were displayed on a computer screen. Movements were out and back from a central starting point to one of eight radial targets. The following specific tasks were performed.

CCW. The eight targets appeared in a predictable counterclockwise order. Subjects had to reach for the target, hitting it within a time window of 200 milliseconds before and after the tone. ${ }^{5}$ During PET, this task was used as a kinematically equivalent reference condition for the motor sequence learning task. ${ }^{6,8}$ A resting sensory controlled state served as the reference condition for quantifying motor activation responses during the performance of the CCW task. ${ }^{5,6,9}$

RAN. Targets were presented in a pseudorandomized and unpredictable order. Subjects were required to reach the targets as soon as possible after the target appearance but avoiding target anticipation. ${ }^{6}$ This task was used to determine choice reaction time distributions and their floor for each subject and to assess concurrent motor performance.

SEQ. The eight targets appeared in an unknown order that was repeated over the 90-second trial block. Subjects were informed that a sequence was to be presented; they were instructed to learn the sequence order while reaching for the targets, to anticipate successive targets, and to reach each target in synchrony with the tone. ${ }^{6,8}$ At the end of the block, they reported the order of the sequence verbally. This task was kinematically equivalent to CCW and was used to assess learning performance. Sequences were different each trial run.

\section{Task Performance}

The following measures were computed for each movement in each of the motor tasks ${ }^{5,6}$ : (1) Spatial error: the shortest distance of the end point from the center of the target; (2) directional error at the peak velocity: the difference between the direction of the vector from the starting point to the target and that of the vector from the starting point to the movement peak velocity; (3) movement time: the time from movement onset to the reversal point; (4) onset time: the time from target and tone presentation to movement onset. Negative values indicate responses that initiated before the tone. In each task, we computed means and variances across the entire trial block, as well as for the complete cycle of eight movements. Repeated measures analysis of variance (RMANOVA) with post hoc comparisons was performed on each of the analytical variables to assess the effects of groups, cycles, and their interaction. These analyses were considered significant for $p$ value less than 0.05 .

In addition, for the sequence learning task, we computed the number of correct movements initiated before the floor onset time in RAN. These movements reflect anticipation and successful retrieval of previously acquired targets. ${ }^{6,8}$ The sum of all correctly anticipated targets in each trial block was defined as the global retrieval index. The number of total correct target locations verbally reported by each subject at the end of a trial block (from 0 -unawareness of a repeating sequence to 8-complete correct sequence) represented a declarative score, an additional measure of the explicit learning achieved. Global learning indices and declarative scores were compared across subjects by correlation analysis.

\section{Imaging}

Seven of the nonmanifesting DYT1 carriers and seven of the control subjects (age, $50.5 \pm 12.2$ years) performed the motor learning (SEQ) and execution (CCW) tasks while undergoing PET imaging. All subjects fasted overnight before the imaging experiments. $\mathrm{H}_{2}{ }^{15} \mathrm{O}$ PET imaging was performed using the GE Advance tomograph at North Shore University Hospital in three-dimensional mode according to procedures as described in detail elsewhere. ${ }^{6}$ During the PET session, each subject was scanned while performing the two tasks in randomized order with the dominant right arm. ${ }^{8,9}$ Each task was repeated twice. For SEQ, different sequences were used for each scan; psychophysical recording of learning performance was acquired for every run.

\section{Data Analysis}

Data processing was performed using SPM99 software (Wellcome Department of Cognitive Neurology, London, UK). Group comparison of regional cerebral blood flow (rCBF) during motor performance (CCW) was performed by generating SPM $\{t\}$ maps. To identify voxels that were activated differently in the two groups during learning, we used a two-factor ANOVA that included both groups (DYT1 and controls) and conditions (SEQ and CCW). Areas with increased activation in DYT1 carriers relative to controls were detected in the model $\left(\mathrm{DYT} 1_{\mathrm{SEQ}}, \mathrm{DYT} 1_{\mathrm{CCW}}\right.$, Controls $\mathrm{SEQ}_{\mathrm{SE}}$ Controls $\left.s_{\mathrm{CCW}}\right)$ by specifying a contrast of $(1,-1,-1,1)$; areas with relatively reduced activation in the DYT1 group were detected by specifying a contrast of $(-1,1,1,-1)$. In all analyses, activations were considered significant at a threshold of $p=0.05$, corrected for multiple comparisons. In addition, we reported activations at $p=0.001$ at peak voxel, if related to prior hypotheses. Coordinates were reported in the standard anatomical space developed at the Montreal Neurological Institute. ${ }^{10}$ Cerebellar localization was performed according to the atlas of Schmahmann and colleagues. $^{11}$

To determine whether group differences at the reported voxels were attributed to the reference scans, we performed post hoc comparisons of rCBF within each condition. These effects were considered meaningful for $p<0.05$ (Student's $t$-test, two tailed). 


\section{Results}

\section{General Motor Performance}

There were no significant differences between DYT1 carriers and control subjects in the performance of CCW and RAN (Fig 1). In CCW, the two groups did not differ in spatial error (mean \pm SD: DYT1: $0.29 \pm$ 0.12 ; controls: $0.22 \pm 0.10 \mathrm{~cm} ; p=0.17)$, movement time (DYT1: $417.2 \pm 90.8$; controls: $416.0 \pm 82.4$ milliseconds; $p=0.97$ ), and onset time (DYT1: $-451.8 \pm 117.3$; controls: $-422.6 \pm 123.6$ milliseconds; $p=0.56$ ). Similar results were obtained with RAN. In this task, the two groups did not differ in mean reaction time (DYT1: $245.5 \pm 44.6$; controls: $253.9 \pm 51.8$ milliseconds; $p=0.67$ ) or movement time (DYT1: $334.5 \pm 61.6$; controls: $312.3 \pm 47.2$ milliseconds; $p=0.33$ ). Floor reaction times were also similar for the two groups (DYT1: $145.1 \pm 11.9$; controls: $151.7 \pm 19.8$ milliseconds; $p=0.33$ ).

\section{Sequence Learning}

During SEQ, DYT1 carriers and controls had similar movement time (mean \pm SD: DYT1: $344.1 \pm 59.7$; controls: $348.4 \pm 47.9$ milliseconds, $p=0.90$ ) and

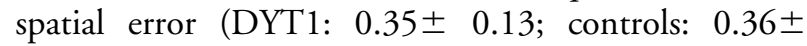
$0.09 \mathrm{~cm} ; p=0.98)$. However, sequence learning was impaired in DYT1 carriers compared with age-matched controls. The time course of onset time for correct movements in each group is presented in Figure 2A. In the control group, mean onset time decreased below the RAN reaction time floor by the second cycle, became negative by the fifth cycle, and reached a plateau by the seventh cycle. In contrast, onset time in DYT1 carriers did not on average cross the RAN reaction time floor. RMANOVA showed a significant main ef-

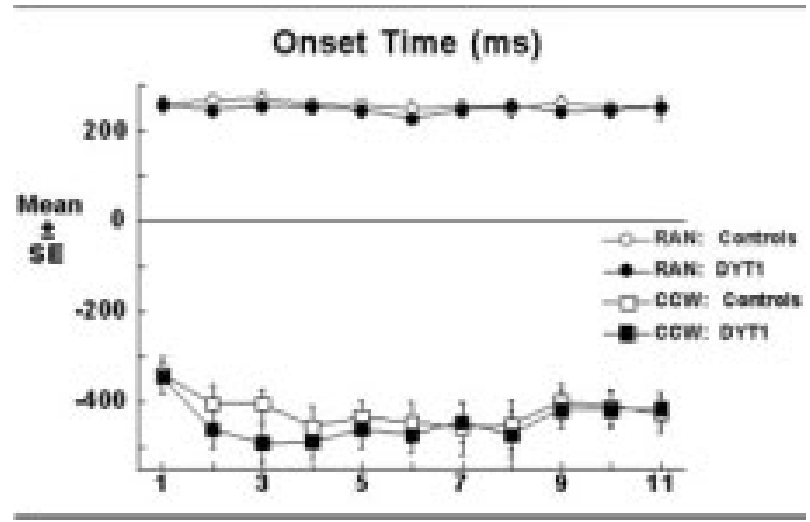

Fig 1. Mean onset times during the execution of the motor tasks CCW (squares) and RAN (circles) in nonmanifesting DYT1 carriers (filled symbols) and age-matched control subjects (open symbols). Values representing the average of two runs in each subject are plotted as a function of cycles (see text). The two groups did not differ for onset time or other kinematic parameters during either task (bars indicate SE). $m s=$ millisecond .
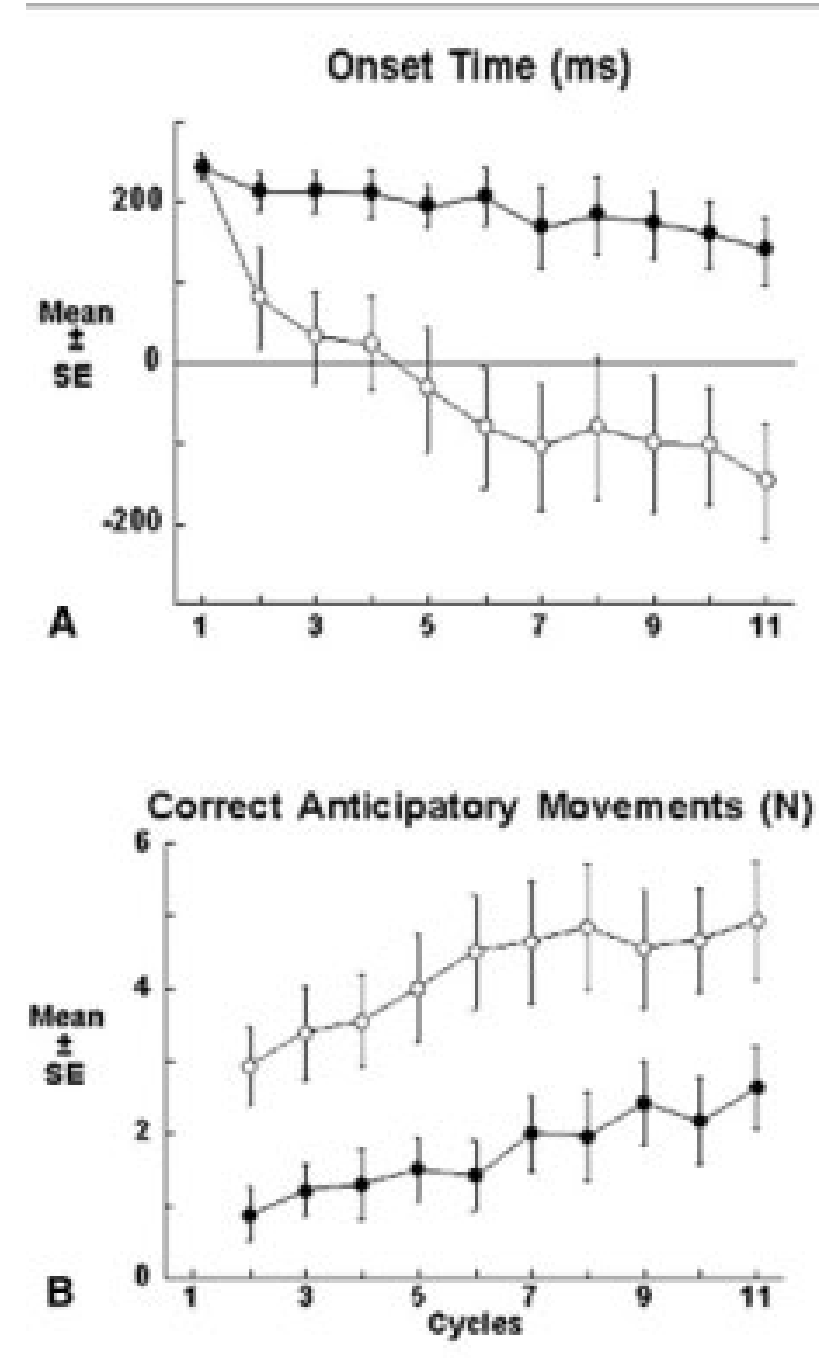

Fig 2. Mean onset times $(A)$ and number of correctly anticipated movements $(B)$ during motor sequence learning in nonmanifesting DYT1 carriers (filled circles) and controls (open circles). Values are plotted as a function of cycles (see text). Both variables changed over time, indicating target prediction and learning. ${ }^{6}$ Task performance in the 90-second epoch was significantly lower in the DYT1 cohort $(\mathrm{p}<0.01)$.

fect of groups $(F[1,220]=8.91, p<0.007)$ and cycles $(\mathrm{F}[10,220]=17.12, p<0.0001)$, as well as a significant interaction between groups and cycles $(\mathrm{F}[10,220]=6.72, p=0.001)$, indicating that the time course of learning was different for the two subject cohorts.

Target retrieval increased progressively across cycles in both groups, although differently (see Fig 2B). RMANOVA identified a significant main effect of both cycles $(\mathrm{F}[9,198]=7.2, p<0.0001)$ and groups $(\mathrm{F}[1,198]=8.60, p<0.008)$, and no significant interaction between group and cycles $(\mathrm{F}[10,220]=0.84$, $p=0.58)$. The global retrieval index across cycles was $41.92 \pm 24.39$ (mean \pm SD) in controls and $17.46 \pm$ 
15.50 in DYT1 gene carriers $(p<0.008)$. The declarative score was also significantly lower in the DYT1 carriers than in the control subjects $(2.50 \pm 1.89$ and $5.92 \pm 2.12$, respectively; $p<0.0005$ ). In both groups, this verbal score was highly correlated with the global retrieval index measured during SEQ task performance (DYT1: $R^{2}=0.69, p<0.001$; controls: $\left.\mathrm{R}^{2}=0.74, p<0.0005\right)$.

\section{Imaging}

GENERAL MOTOR PERFORMANCE. In DYT1 carriers, the motor reference task (CCW) was associated with activation of the left sensorimotor and premotor cortices (SMC and PMC, respectively), ventral thalamus, and the right SMA and a small area of the SMC, inferior occipital association cortex (BA 19), and posterior cerebellum. A small activation focus also was identified within the right SMC. The same regions were identified during motor activation in the control group. Comparison of the two groups (Table 1; Fig 3) showed a relative increase in motor activation in the DYT1 cohort that was localized to the right SMA and the left lateral PMC and inferior parietal cortex (BA 40). In contrast, a relative reduction in motor activation was present in DYT1 carriers in the left posterior medial cerebellum.

SEQUENCE LEARNING. In DYT1 carriers, sequence learning (SEQ - CCW) was associated with activation of the right dorsal PMC, pre-SMA, and the visual association cortices (BA 18, 19). This pattern of learning-related regional activation was present in the control group. However, compared with controls, DYT1 carriers exhibited increased activation during learning in the right pre-SMA, in occipital association cortex (BA 19) and cerebellar hemisphere, and in the left ventral prefrontal cortex (BA 11) (Table 2; Fig 4).

Table 1. Brain Regions in Which DYT1 Carriers and Control Subjects Differed in Regional Cerebral Blood Flow during Motor Execution

\begin{tabular}{|c|c|c|c|c|}
\hline \multirow[b]{2}{*}{ Region } & \multicolumn{3}{|c|}{$\begin{array}{l}\text { Standard Space } \\
\text { Coordinate }\end{array}$} & \multirow[b]{2}{*}{$Z_{\max }$} \\
\hline & $x$ & $y$ & $z$ & \\
\hline \multicolumn{5}{|l|}{ Increased activation } \\
\hline Right SMA (BA 6) & 10 & -18 & 72 & 4.07 \\
\hline $\begin{array}{l}\text { Left inferior parietal lobe } \\
\text { (BA 40) }\end{array}$ & -58 & -56 & 32 & 3.72 \\
\hline Left lateral PMC (BA 6) & -30 & 8 & 52 & 3.38 \\
\hline \multicolumn{5}{|l|}{ Decreased activation } \\
\hline Left cerebellum, lobulus IX & -2 & -54 & -34 & 4.45 \\
\hline
\end{tabular}
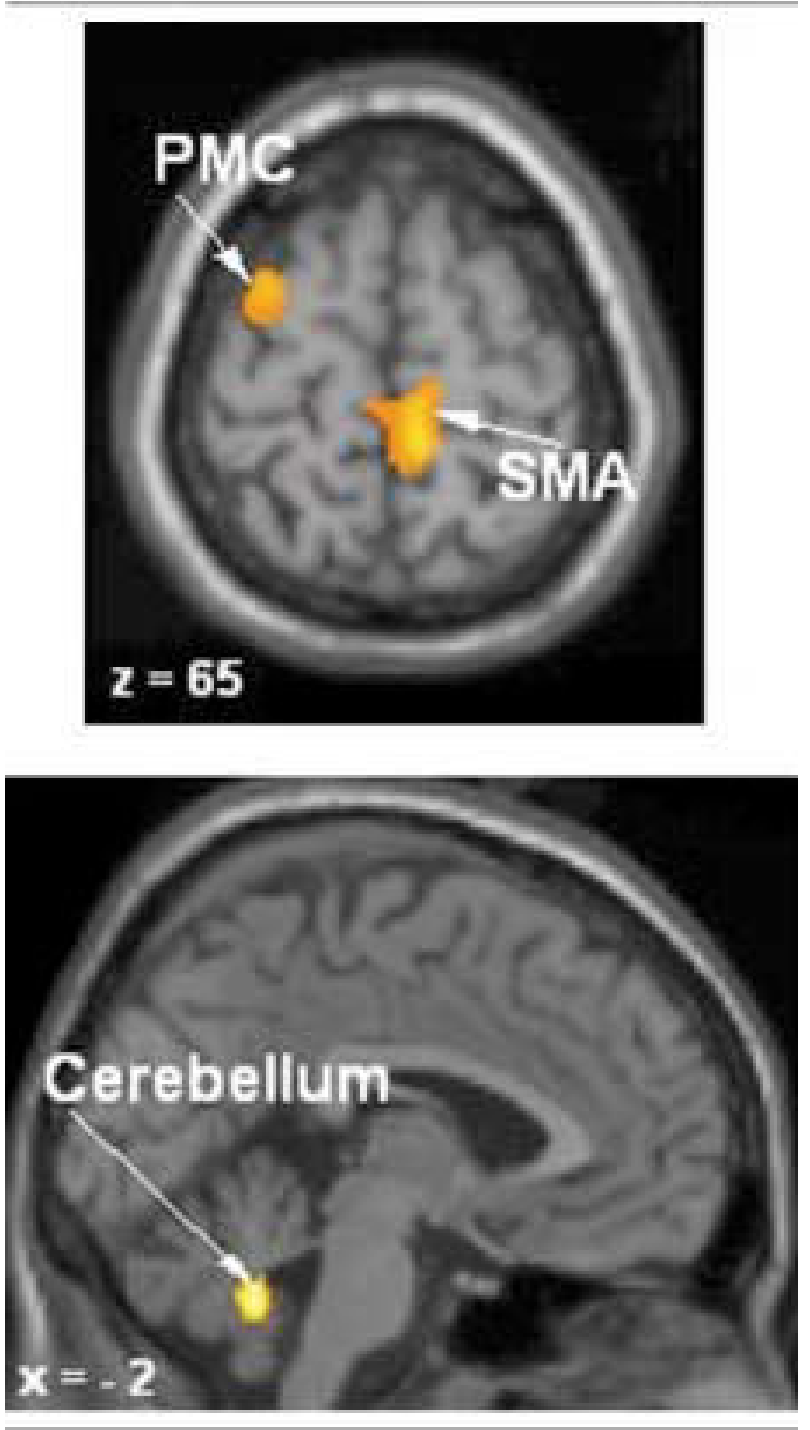

Fig 3. Brain regions in which regional cerebral blood flow recorded during motor execution differed in nonmanifesting DYT1 carriers and age-matched control subjects (see text). The right supplementary motor area (SMA) and left premotor cortex (PMC) (top) were overactive in gene carriers relative to controls. In contrast, motor activation in the posterior medial cerebellum (bottom) was relatively impaired in these subjects. The color stripe represents $T$ values thresholded at 3.55, $\mathrm{p}<$ 0.001 .

In contrast, gene carriers exhibited subnormal activation in the posterior aspect of the left cingulate gyrus (BA 24). Post hoc testing confirmed that in these regions group differences in blood flow were not referable to the CCW reference condition $(p>0.1$ at each locus).

\section{Discussion}

This study presents evidence of impaired motor sequence learning in clinically unaffected carriers of the DYT1 dystonia mutation. Although general motor per- 
Table 2. Brain Regions in Which DYT1 Carriers and Control Subjects Differed in Regional Activation during Motor Sequence Learning

\begin{tabular}{|c|c|c|c|c|}
\hline \multirow[b]{2}{*}{ Region } & \multicolumn{3}{|c|}{$\begin{array}{l}\text { Standard Space } \\
\text { Coordinate }\end{array}$} & \multirow[b]{2}{*}{$Z_{\max }$} \\
\hline & $x$ & $y$ & $z$ & \\
\hline \multicolumn{5}{|l|}{ Increased activation } \\
\hline Right pre-SMA (BA 6) & 10 & 8 & 54 & 3.54 \\
\hline Right cuneus (BA 19) & 30 & -84 & 12 & 3.90 \\
\hline $\begin{array}{l}\text { Right cerebellar hemisphere, } \\
\text { lobulus IV }\end{array}$ & 16 & -32 & -28 & 4.03 \\
\hline $\begin{array}{l}\text { Left ventral prefrontal cor- } \\
\text { tex (BA 11) }\end{array}$ & -14 & 46 & -22 & 3.24 \\
\hline \multicolumn{5}{|l|}{ Decreased activation } \\
\hline $\begin{array}{l}\text { Left cingulate gyrus } \\
\quad(\text { BA 24) }\end{array}$ & -10 & -10 & 40 & 4.22 \\
\hline
\end{tabular}

a $p<0.05$, corrected for multiple comparisons. The other values are significant at $p=0.001$ (uncorrected).

$\mathrm{SMA}=$ supplementary motor area; $\mathrm{PMC}=$ premotor cortex.

formance in gene carriers was similar to controls, regional activation during movement was abnormally increased in auxiliary motor areas. In contrast, DYT1 carriers exhibited a defect in the learning of sequential movements, which was associated with abnormal activation responses in prefrontal cortex and cerebellum. These findings suggest that even in the absence of dystonia, DYT1 carriers engage anatomical/functional neural pathways differently than control subjects.

\section{Motor Performance}

Reaching performance of the DYT1 and control groups did not differ for timing or spatial accuracy, contrasting with the abnormalities reported in affected dystonia patients. ${ }^{12}$ Despite the kinematic equivalence of CCW and RAN, these motor tasks have different temporal requirements. CCW is a timed-response task, which allows for the evaluation of both spatial and temporal movement accuracy. ${ }^{5,9}$ In this task, subjects are instructed to reach the targets in synchrony with repeating tones, requiring them to program movements based on an accurate prediction of both the locus and time of the upcoming target, as well as the duration of movement. This experimental paradigm promotes the development of anticipation and the internal generation of movements. In contrast, reaction time paradigms such as RAN require neural processing for stimulus identification and for response selection and programming within a brief time interval. Thus, movements produced in RAN can be considered externally generated. Furthermore, because of the rhythmical target presentation in RAN, information processing and movement execution for each target must be conducted in less than a second for successful performance. Our results indicate that the production of both internally and externally generated movements, as well as stimulus information processing, movement programming, and target anticipation, is preserved in nonmanifesting DYT1 carriers.

Despite normal trajectory control in all the tasks, the learning of new motor sequences is significantly slower in nonmanifesting DYT1 gene carriers compared with age-matched controls. Our sequence learning paradigm promotes and measures explicit and conscious acquisition of target order. In this task, subjects are informed in advance of the presence of a sequence and are instructed to anticipate target appearance during learning. Indeed, the retrieval index extracted from the motor performance data is highly correlated with the subject's verbal report, which reflects the degree of awareness and explicit learning. We found that both learning indices are drastically reduced in DYT1 gene carriers relative to control subjects. This deficit cannot be simply attributed to an underlying motor deficit: movement time and spatial accuracy in DYT1 carriers was normal in all motor tasks. Moreover, we have reported that these subjects have difficulty in learning simple sequences visually or by trial and error, whereas their adaptation to a rotated visual display is normal. ${ }^{13}$ Successful performance in all our sequence learning tasks is likely to depend on spatial working memory to acquire and retrieve sequences, to compare successive targets to those presented previously. Thus, depleted working memory resources may be responsible for the substantial impairment in sequence learning that was detected in DYT1 carriers. Nonetheless, there was no behavioral evidence of a broader cognitive deficit, consistent with the normal cortical histopathology associated with primary dystonia. ${ }^{14}$

In this study, the DYT1 group was compared with a demographically more diverse control cohort. Although the estimated prevalence of this mutation in the control population is likely to be quite low $(<0.3 \%)$, we cannot exclude the possibility that factors apart from the DYT1 genotype contributed to the observed behavioral differences. Another consideration relates to subject age. DYT1 dystonia usually manifests during childhood or early adulthood. To increase the likelihood of selecting true nonpenetrant (as opposed to presymptomatic) participants, we recruited neurologically normal DYT1 carriers older than age 30 years. ${ }^{3}$ Recently, however, the onset of clinical symptoms was reported in a 64-year-old DYT1 carrier. Nonetheless, the late appearance of dystonia in nonmanifesting gene carriers can be considered rare and is unlikely to have an impact on the reported psychophysical observations.

\section{Brain Activation}

The general pattern of motor activation during CCW was similar in DYT1 carriers and control subjects., ${ }^{5,6,9}$ Nonetheless, despite comparable task performance, the 

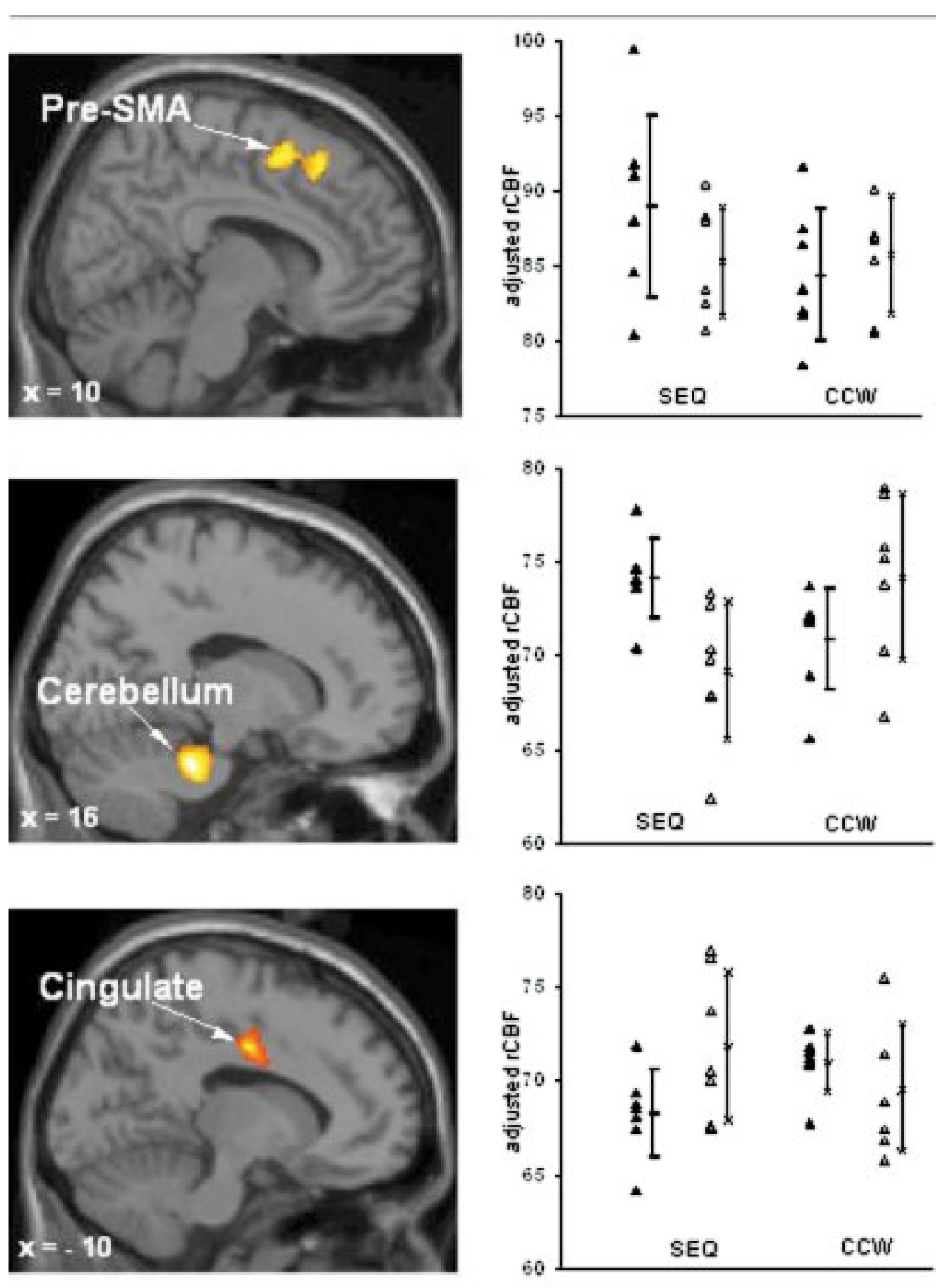

Fig 4. Brain regions in which activation differences recorded during motor sequence learning differed in DYT1 carriers (filled triangles) and controls (open triangles). In gene carriers, increased learning-related activation was present in the right pre-supplementary motor area (SMA) (top) and in the anterior lateral cerebellum (middle). In contrast, activation in the left cingulate gyrus (bottom) was decreased relative to controls. Scatterplots to the right of the corresponding slices represent adjusted regional cerebral blood flow ( $r C B F$ ) during sequence learning (SEQ) and during the motor reference task (CCW) in the voxel of the maximum group difference. In all these areas, $r C B F$ differences during CCW performance were not statistically significant (see text). The color stripe represents $T$ values thresholded at 3.05, $\mathrm{p}<0.005$. 
gene carriers displayed enhanced activation in premotor areas and in multimodal parietal association cortex. Overactivation of SMA has been reported in affected idiopathic dystonia patients performing freely chosen joystick movements. ${ }^{15-18}$ The presence of this abnormality in nonmanifesting DYT1 carriers raises the possibility of increased excitability of premotor cortex as a physiological trait of genetically susceptible individuals. Indeed, the activation findings are consistent with our previous observations of relative SMA hypermetabolism in DYT1 carriers scanned at rest. ${ }^{3,4}$ We cannot, however, exclude the possibility that the increase in SMA activity during motor performance was caused by abnormal muscle force, ${ }^{19}$ perhaps related to increased levels of co-contraction. Nonetheless, SMA overactivation also has been reported in sensory deprivation after amputation, ${ }^{20}$ consistent with the possibility of abnormal sensory processing in dystonia. ${ }^{21}$ The increase in parietal activation during $\mathrm{CCW}$ also may be compatible with this notion. Underactivation of the contralateral $\mathrm{SMC}$, a prominent feature of motor execution in clinically manifesting dystonia patients, ${ }^{15-17}$ is not present in the unaffected gene carriers. The presence of abnormal SMC activation, caused either by a primary lesion or by abnormal thalamic input, may be critical for the development of symptoms in manifesting DYT1 carriers.

Overactivation of the basal ganglia and cerebellum during joystick movement has been described consistently in torsion dystonia. ${ }^{15-17}$ Despite the presence of resting network-related hypermetabolism of the lentiform nuclei in nonmanifesting carriers of several primary torsion dystonia mutations, ${ }^{3,4}$ we did not identify abnormal motor activation responses in the basal ganglia of the reported DYT1 carriers. This may be attributable to the small magnitude of putamen and globus pallidus activation recorded during CCW performance, ${ }^{5,6}$ making group differences in these areas comparatively difficult to detect. Alternatively, the presence of localized metabolic increases in the resting state in these structures may limit their capacity to activate during a simple motor task.

In contrast, motor activation responses were reduced in the posterior-medial cerebellum of nonmanifesting DYT1 carriers. This finding contrasts with affected dystonia patients in whom relative increases in cerebellar activation have been described during joystick movement. ${ }^{17}$ As previously suggested, ${ }^{3}$ reduced motor activation in the cerebellum of DYT1 carriers may relate to the deposition of mutant torsion A protein in this region..$^{22,23}$ It is also conceivable that diminished cerebellar activation during motor execution is a reflection of impaired proprioceptive feedback during coordinated movements, ${ }^{24}$ as has been demonstrated in focal dystonia. ${ }^{25}$ It would be of interest to know if similar abnormalities are present in nonmanifesting carriers of dystonia mutations, and whether increased SMA activation occurs in compensation for this potential functional defect.

Despite the presence of a major impairment of sequence learning in DYT1 carriers, the overall regional activation pattern was similar to that of control subjects, as well as independent cohorts of volunteers scanned while performing this and related tasks., $5,8,26$ However, during sequence learning, DYT1 carriers exhibited increased activation in several of the brain regions activated by controls during task performance. Comparison of the two groups showed enhanced rCBF responses in the left prefrontal cortex, and in the right pre-SMA, occipital association cortex, and lateral cerebellum. Overactivation of these regions during learning may represent a means of compensating for striatal dysfunction, as suggested previously. ${ }^{6}$ Nonetheless, this physiological response is inadequate to achieve a normal level of learning performance in the gene carriers.

The role of the anterior-lateral cerebellar cortex in the motor sequence learning process warrants additional comment. This area projects to the dorsolateral prefrontal $\operatorname{cortex}^{27}$ and contributes to anatomical/functional networks involved in attention and the learning of cognitive routines. It has been proposed that lesions in areas of the cerebellum that project to prefrontal cortex can mimic the cognitive deficits that are typical of frontal lobe damage. ${ }^{28,29}$ Although learning-related dorsolateral prefrontal cortex activation is not significantly altered in DYT1 carriers, this region is associated with cerebellar activity through its distinctive pattern of connectivity. Indeed, preliminary network analysis of the DYT1 imaging data has shown prominent cerebellar-prefrontal activation during learning, as compared with the normal pattern of striatocortical regional covariation. The relative roles of the cerebellum and striatum in predicting learning performance in DYT1 carriers can be determined through the analysis of additional subjects.

In addition to areas of functional overactivity in DYT1 carriers, we identified an area of reduced learning-related activation in the posterior aspect of the left cingulate gyrus (BA 24). This finding may be relevant to the learning defect by virtue of its proximity to the posterior cingulate cortex, an area related to cued spatial attention. ${ }^{30}$ A functional deficit in this region may be consistent with the difficulty in switching between movements that has been reported in affected dystonia patients. ${ }^{31}$

The mechanism of impaired motor sequence learning and altered brain activation responses in nonmanifesting carriers of the DYT1 mutation is unknown. Recent electrophysiological studies have attributed dystonia to an altered pattern of pallidal output with burst firing and highly irregular interspike frequencies. ${ }^{32,33}$ If present to a lesser degree in nonmanifesting dystonia 
gene carriers, this subcortical noise source may interfere with the functioning of corticostriatopallidothalamocortical and related pathways, and with associated aspects of motor behavior including the learning of sequences. Nonetheless, the possibility of an inherent genetically mediated abnormality in frontostriatal connectivity cannot be excluded. Further imaging studies of functional/anatomical connectivity in dystonia gene carriers may shed light on these issues.

This work was supported by the National Institutes of Health (NIH RO1 NS 37564, D.E.; NIH KO8 NS 01961, M.-F.G.; NIH K24 NS 02101, D.E.) and the Dystonia Medical Research Foundation (D.E.).

We thank Dr T. Chaly for radiochemistry support, and C. Edwards and S. Su for editorial assistance. We acknowledge the valuable technical support provided by Dr A. Belakhleff and C. Margouleff.

\section{References}

1. Nemeth AH. The genetics of primary dystonias and related disorders. Brain 2002;125:695-721.

2. Klein C, Ozelius LJ. Dystonia: clinical features, genetics, and treatment. Curr Opin Neurol 2002;15:491-497.

3. Eidelberg D, Moeller JR, Antonini A, et al. Functional brain networks in DYT1 dystonia. Ann Neurol 1998;44:303-312.

4. Trost M, Carbon M, Edwards C, et al. Primary dystonia: is abnormal functional brain architecture linked to genotype? Ann Neurol 2002;52:853-856.

5. Ghilardi M, Ghez C, Dhawan V, et al. Patterns of regional brain activation associated with different forms of motor learning. Brain Res Bull 2000;14:127-145.

6. Nakamura T, Ghilardi MF, Mentis M, et al. Functional networks in motor sequence learning: abnormal topographies in Parkinson's disease. Hum Brain Mapp 2001;12:42-60.

7. Wichmann T, DeLong MR. Models of basal ganglia function and pathophysiology of movement disorders. Neurosurg Clin $\mathrm{N}$ Am 1998;9:223-236.

8. Fukuda M, Ghilardi MF, Carbon M, et al. Pallidal stimulation for parkinsonism: improved brain activation during sequence learning. Ann Neurol 2002;52:144-152.

9. Fukuda M, Ghilardi MF, Mentis MJ, et al. Functional correlates of pallidal stimulation for Parkinson's disease. Ann Neurol 2001;124:1601-1609.

10. Collins L, Neelin P, Peters T, Evans A. Automatic 3-D intersubject registration of MR volumetric data in standardized Talairach space. J. Comput Assist Tomogr 1994;18:192-205.

11. Schmahmann JD, Doyon J, Toga AW, et al. MRI atlas of the human cerebellum. San Diego: Academic Press, 2000.

12. Jahanshahi M, Rowe J, Fuller R. Impairment of movement initiation and execution but not preparation in idiopathic dystonia. Exp Brain Res 2001;140:460-468.

13. Ghilardi MF, Ghez C, Eidelberg D. Visuospatial learning may be impaired in non-manifesting carriers of the DYT1 mutation. Neurology 1999;52:A516.
14. Hedreen JC, Zweig RM, DeLong MR, et al. Primary dystonias: a review of the pathology and suggestions for new directions of study. Adv Neurol 1988;50:123-132.

15. Ceballos-Baumann AO, Passingham RE, Warner $\mathrm{T}$, et al. Overactive prefrontal and underactive motor cortical areas in idiopathic dystonia. Ann Neurol 1995;37:363-372.

16. Ceballos-Baumann AO, Brooks DJ. Basal ganglia function and dysfunction revealed by PET activation studies. Adv Neurol 1997;74:127-139.

17. Playford ED, Passingham RE, Marsden CD, Brooks DJ. Increased activation of frontal areas during arm movement in idiopathic torsion dystonia. Mov Disord 1998;13:309-318.

18. Kumar R, Dagher A, Hutchison WD, et al. Globus pallidus deep brain stimulation for generalized dystonia: clinical and PET investigation. Neurology 1999;53:871-874.

19. Cramer SC, Weisskof RM, Schaechter JD, et al. Motor cortex activation is related to force of squeezing. Hum Brain Mapp 2002;16:197-205.

20. Dettmers C, Adler T, Rzanny R, et al. Increased excitability in the primary motor cortex and supplementary motor area in patients with phantom limb pain after upper limb amputation. Neurosci Lett 2001;307:109-112.

21. Kaji R, Shibasaki H, Kimura J. Writer's cramp: a disorder of motor subroutine? Ann Neurol 1995;38:862-868.

22. Augood SJ, Martin DM, Ozelius LJ, et al. Distribution of the mRNAs encoding torsin A and torsinB in the normal adult human brain. Ann Neurol 1999;46:761-769.

23. Konokova M, Huynh DP, Yong W, Pulst SM. Cellular distribution of torsin $\mathrm{A}$ and torsin $\mathrm{B}$ in normal human brain. Arch Neurol 2001;58:921-927.

24. Ramnani N, Toni I, Passingham RE, Haggard P. The cerebellum and parietal cortex play a specific role in coordination: a PET study. Neuroimage 2001;14:899-911.

25. Sigglekow S, Kossev A, Moll C, et al. Impaired sensorimotor integration in cervical dystonia: a study using transcranial magnetic stimulation and muscle vibration. J Clin Neurophysiol 2002;19:232-239.

26. Jueptner M, Weiller C. A review of differences between basal ganglia and cerebellar control of movements as revealed by functional imaging studies. Brain 1998;121:1437-1449.

27. Middleton FA, Strick PL. Cerebellar projections to the prefrontal cortex of the primate. J Neurosci 2001;21:700-712.

28. Fiez JA, Petersen SE, Cheney MK, Raichle ME. Impaired nonmotor learning and error detection associated with cerebellar damage. A single case study. Brain 1992;115:155-178.

29. Fiez JA. Cerebellar contributions to cognition. Neuron 1996; 16:13-15.

30. Mesulam MM, Nobre AC, Kim YH, et al. Heterogeneity of cingulate contributions to spatial attention. Neuroimage 2001; 13:1065-1072.

31. Agostino R, Berardelli A, Formica A, et al. Sequential arm movements in patients with Parkinson's disease, Huntington's disease, and dystonia. Brain 1992;115:1481-1495.

32. Gernert M, Bennay M, Fedrowitz M, et al. Altered discharge pattern of basal ganglia output neurons in an animal model of idiopathic dystonia. J Neurosci 2002;22:7244-7253.

33. Vitek JL. Pathophysiology of dystonia: a neuronal model. Mov Disord 2002;17(suppl 3):S49-S62. 